\title{
Association of subclinical psychosis with suicidal ideation : A twin study
}

\section{O'Hare, Kirstie J. M.}

2020-09

O'Hare , K J M , Korhonen , T, Latvala , A , Kaprio , J \& Linscott , R J 2020 , ' Association of subclinical psychosis with suicidal ideation : A twin study ', Schizophrenia Research, vol. 223 , pp. 173-178 . https://doi.org/10.1016/j.schres.2020.07.010

http://hdl.handle.net/10138/338262

https://doi.org/10.1016/j.schres.2020.07.010

cc_by_nc_nd

acceptedVersion

Downloaded from Helda, University of Helsinki institutional repository.

This is an electronic reprint of the original article.

This reprint may differ from the original in pagination and typographic detail.

Please cite the original version. 


\section{Association of Subclinical Psychosis with Suicidal Ideation: A Twin Study}

Running title: Subclinical Psychosis and Suicidal Ideation

Kirstie J. M. O’Hare, ${ }^{1,}$, Tellervo Korhonen ${ }^{2}$, Antti Latvala ${ }^{3}$, Jaakko Kaprio ${ }^{2,4}$, Richard J. Linscott $^{1}$

Affiliations:

${ }^{1}$ Department of Psychology, University of Otago, Dunedin, New Zealand

${ }^{2}$ Institute for Molecular Medicine Finland, University of Helsinki, Helsinki, Finland

${ }^{3}$ Institute of Criminology and Legal Policy, University of Helsinki, Helsinki, Finland

${ }^{4}$ Department of Public Health, University of Helsinki, Helsinki, Finland

* Corresponding author at: Department of Psychology, University of Otago, P. O. Box 56,

Dunedin 9054, New Zealand. Tel.: +64 3479 5689; fax: +64 34798335.

E-mail address: kirstie.ohare@gmail.com

Word Counts

Abstract: 221

Text body: 4002 


\begin{abstract}
Subclinical psychosis, including schizotypal indicators and psychotic experiences, predicts future suicidal ideation. This relationship may reflect unmeasured confounding from environmental factors, genetic factors, or both. We used a genetically-informative twin design to understand if the association between subclinical psychosis and suicidal ideation is independent of shared genetic and environmental factors. We analysed cross-sectional associations of age-22 self-reported subclinical psychosis (positive, negative, and disorganised features) with suicidal ideation in twins participating in the FinnTwin12 study (maximum $n=1213$ ). Then, we analysed the reverse association of age-14 suicidal ideation with age-22 subclinical psychosis. Associations were studied first among individuals and then within monozygotic (MZ) and dizygotic (DZ) pairs. Individual-level analyses showed that all subclinical psychosis factors were associated with suicidal ideation. In within-pair analyses, estimates of associations were lower for MZ pairs than DZ pairs, except for the negative schizotypy-suicidal ideation association where estimates were consistent across individuallevel and within-pair analyses. Findings provide evidence that the association between negative features and suicide ideation is not explained by familial factors and may be causal, though the possibility of confounding by individual-specific environmental factors and reverse causation cannot be ruled out. The relationships of positive and disorganised subclinical psychosis features with suicidal ideation cannot be explained by confounding due to environmental factors shared between siblings, but their associations may be due to shared genetic factors.
\end{abstract}

Key words: Psychotic Experiences, Schizotypy, Suicide, Twins, Genetics, Causality 


\section{Introduction}

Suicide is a major public health problem, with over 800,000 people taking their own lives each year (World Health Organization, 2014). Suicidal ideation and behaviours (SIB) are particularly prominent in adolescents and young adults, and represent a leading cause of death in this age group (Hawton et al., 2012). Despite a large increase in the number of published studies on suicide risk factors in the past 50 years, predictive ability of these factors has not improved (Franklin et al., 2017) and there is a lack of progress in understanding risk factors that generate suicidal thoughts and behaviours (Glenn et al., 2017; Prinstein, 2008). The past decade has seen accumulating evidence that subclinical psychosis predicts SIB (Taylor et al., 2015; Honings et al., 2016; Hielscher et al., 2018; Yates et al., 2019).

Diverse terms are used to describe subclinical psychosis, including psychotic or psychotic-like experiences, delusion- and hallucination-like experiences, psychosisproneness, psychoticism, and schizotypy. Although there are not clear measurement boundaries among these, distinction can be drawn between two main but overlapping categories, namely isolated positive features (hallucinations and delusions) known as psychotic experiences, and a broader set of positive, negative, and disorganisation traits known as schizotypy. Schizotypy is thought of as a multidimensional construct (Raine et al., 1994), and different components are heterogenous in that they have differential relationships with environmental and genetic factors, (e.g. Esterberg et al., 2009; Jones et al., 2018; Morton et al., 2016). Most research on the subclinical psychosis-SIB relationship has focused on psychotic experiences, but there is evidence that interpersonal or negative (Jahn et al., 2016) and disorganised (Teraishi et al., 2014) features also relate to suicidality.

Whether this schizotypy-SIB relationship is spurious or causal is unclear. Some have suggested that the schizotypy-SIB relationship can be explained as arising from the common influence of environmental risk factors (DeVylder et al., 2015; Jahn et al., 2016), but there is 
no consensus on which environmental factors are confounding this relationship (Hielscher et al., 2018). Another potential explanation for the relationship is that schizotypy and SIB are spuriously associated because they share some of the same underlying genetic vulnerability. Previous twin studies have indicated that schizotypy is a moderately heritable trait, with around $40 \%$ to $60 \%$ of the variance attributable to genetic factors (Ericson et al., 2011; Lin et al., 2007; Linney et al., 2003). Suicidal ideation is also moderately heritable, with around $40 \%$ of the variance attributable to genetic factors (Brent and Mann, 2005). Currently, there are no studies examining genetic confounding of the schizotypy-SIB relationship.

Examining whether the schizotypy-SIB relationship is the result of confounding by shared genetic factors, environmental factors, or both is difficult due to the observational nature of research on the association. Statistical adjustment for confounding factors is problematic because it relies on complete and accurate identification and measurement of confounders (McGue et al., 2010). Furthermore, statistical control of variables may generate misleading results if the variable is not a true confounder of the relationship of interest (Meehl, 1971). Within-pair twin analyses can be used to identify confounding by shared environment or genetic factors by comparing the association within monozygotic (MZ) and dizygotic (DZ) twin pairs (McGue et al., 2010). Co-twins from both MZ and DZ twin pairs share factors related to their family background (i.e., household, neighbourhood, and parenting factors) as well as other environmental influences, and MZ pairs have the same genomic sequence, while DZ pairs share, on average, $50 \%$ of their segregating genes (McGue et al., 2010). Thus, by relating within-pair differences in schizotypy to within-pair differences in suicide ideation, it can be tested whether the schizotypy-suicidal ideation relationship is present independently of shared environmental and genetic factors.

Though research on the schizotypy-SIB relationship has exclusively focused on the forward association where schizotypy or its components are assumed to cause suicidal 
ideation or behaviours, it is also feasible that the association could be due to reverse causation. For example, it has been proposed that schizotypy or psychotic experiences are a psychological response to the self-directed threat of SIB (Murphy et al., 2018) that or, similarly, that schizotypal indicators could be a psychological adaption to distress occurring from SIB (Hielscher et al., 2018). The relationship between schizotypy and suicidal ideation in general population samples has been tested longitudinally with schizotypy as a predictor of ideation (Yates et al., 2019), but, to our knowledge, not previously with ideation as a predictor of schizotypy. Thus, alongside testing the relationship of schizotypy features with suicidal ideation cross-sectionally, we tested the longitudinal relationship of suicide ideation with subsequent schizotypy.

Our aim was to test whether schizotypy features relate to suicidal ideation in adolescence and young adulthood when controlling for shared genetic and environmental factors. It was hypothesised that schizotypy features predict suicidal ideation in DZ and MZ twin pairs that are discordant for suicidal ideation. Furthermore, the reverse association was tested, that is, whether suicide ideation predicts schizotypy features. If the schizotypy-SIB relationship is due to reverse causation, prior suicidal ideation will be related to subsequent schizotypy at the individual level and in within-pair comparisons of DZ and MZ twins.

\section{Method}

\subsection{Participants and Procedure}

Participants were members of the FinnTwin12 cohort, a population-based longitudinal twin study that began in 1994, using Finland's Population Register Centre to identify Finnish families with twins born from 1983 to 1987. Details about this sample have been reported elsewhere (Kaprio, 2013, 2006). Briefly, at baseline when the twins were aged 11-12, families identified from the register ( $n=2724$ families, $87 \%$ participation rate) were mailed questionnaires. Of these families, $40 \%$ ( $n=1035$ families) were selected for intensive 
assessment. Most of this subsample (72.3\%) was selected at random, but $27.7 \%$ were selected on assumed elevated familial risk of alcoholism, based on parents' (one or both) scores on the 11-item lifetime version of the Malmö-modified Michigan Alcoholism Screening Test (Seppä et al., 1990).

Measures in the present study were obtained when participants were aged 14-years and 22-years on average (range 19.9 to 26.6). At age 14 years, twins were interviewed faceto-face using the Finnish translation of the adolescent version of the Semi-Structured Assessment for the Genetics of Alcoholism (C-SSAGA-A) (Bucholz et al., 1994) by interviewers trained using procedures from the Collaborative Studies on Genetics of Alcoholism (COGA) (Edenberg, 2002). Of invited participants, 90\% participated, resulting in $n=1852$ interviewees. They were invited to participate in a follow-up study in young adulthood, and 1347 SSAGA interviews were completed. At age 22 years, psychiatric assessment occurred in-person in Helsinki $(n=812)$ or via telephone interviews $(n=535)$. Some in-person assessments were completed by telephone due to time restrictions. Participants were administered the SSAGA (Bucholz et al., 1994) and completed self-report questionnaires (in person or mailed at the time of the interview). All participants provided written informed consent. Data collection procedures were approved by the Ethics Committee of Helsinki and the Institutional Review Board of Indiana University, Bloomington, USA.

\subsection{Measures}

Schizotypy was measured at age 22 with the Finnish translation of the Schizotypal Personality Questionnaire-Brief (SPQ-B) (Raine and Benishay, 1995), a 22-item self-report questionnaire designed for the assessment of schizotypal traits. Items require a yes or no response. Recent, comprehensive, cross-national analyses have indicated that the SPQ-B is best described by three factors corresponding to positive (cognitive-perceptual disturbances), negative (interpersonal difficulties), and disorganisation features (Fonseca-Pedrero et al., 
2017), so scores were calculated for each of these three subscales, alongside a total score. Sporadic missing items (fewer than 5 missing ratings) were estimated by using prorated mean subscale scores. Continuous scores were calculated for the three subscales and the total score, then due to the different numbers of items in each subscale, standardised scores were calculated to aid interpretation. Cronbach's alpha coefficient was calculated for the three subscales and total scores.

The Finnish translation of the suicide section of the C-SSAGA-A and the SSAGA was used to assess lifetime suicidal ideation. Lifetime suicidal ideation was classified from responses to a single question, "Have you ever had any thoughts of suicide?", which was administered at age 14 and age 22 interviews. At age 22, the item, "Have you ever tried to commit suicide?" was also administered but was not included in present analyses due to the low number of participants reporting suicide attempts $(n=24)$.

\subsection{Analysis}

We first tested the forward association of suicidal ideation with SPQ-B scores, both obtained at age 22 years. Individual-based analyses (i.e. analyses with no matching of twinpairs, twins are treated as individuals) were conducted using logistic regression with robust standard errors to account for non-independence of observations among twin pairs (Williams, 2000). Analyses were adjusted for age and sex. Then, within-pair associations were estimated using conditional logistic regression among twin pairs discordant for suicidal ideation. Within-pair comparisons determine whether the co-twin that reports higher schizotypy also reports more suicidal ideation (and vice versa), as compared to his or her co-twin. Analyses were conducted separately for DZ pairs and MZ pairs. These three analyses (individual level, DZ within-pair, MZ within-pair) were conducted first with the total SPQ-B score, and then for each of the three subscale scores. The reverse association was then tested by predicting age 22 SPQ-B scores from age 14 suicide ideation. In individual analyses, linear regression 
was used with robust standard errors and age and sex as covariates. Fixed effects linear regression models (Allison, 2009) were used to test within-pair associations, with separate analyses for $\mathrm{DZ}$ and $\mathrm{MZ}$ pairs. These three analyses were conducted with the SPQ-B total score and each of the subscale scores.

One of three patterns of results can be expected to be found (Kujala et al., 2002). If the schizotypy-suicidal ideation relationship is not confounded by shared genetic or environmental factors, the association of schizotypy and suicidal ideation will be the same in the total population as within the matched twin pairs (left model of Figure 1). If the relationship is confounded by shared environmental factors, then the association of schizotypy and suicidal ideation would be expected to be lower in the matched twin pairs (both DZ and MZ) than the total population (centre model of Figure 1). If the relationship is confounded by genetic factors, then the schizotypy-suicidal ideation association will be lower in MZ matched pairs than DZ pairs (right model of Figure 1).

\section{INSERT FIGURE 1 ABOUT HERE}

Additionally, given evidence indicating that schizotypy and suicidal ideation are associated with substance use (Darvishi et al., 2015; Esterberg et al., 2009), the association of random selection (i.e. not selected for increased familial alcoholism risk) with SPQ-B subscales and suicide outcomes was tested using multinomial logistic regression. Intraclass and cross-twin cross-trait correlations were also calculated for the three SPQ-B subscales (positive, negative, and disorganised), and examined for evidence of genetic covariance among the SPQ-B scales. As a sensitivity analysis, the age 22 within-pair analyses were repeated with any ideation (i.e. responded yes to the suicidal ideation item at either age 14 or age 22 assessment) as the outcome.

All analyses were conducted using Stata version 14.2. 


\section{Results}

\subsection{Sample Characteristics}

The sample consisted of 1213 participants who had completed the SSAGA interview and had full data on the SPQ-B at age 22. Of these, 1104 also completed the suicide section of the C-SSAGA-A interview at age 14. SPQ-B subscales or suicide outcomes were not associated with random selection for participation in the study, except for negative schizotypy, which was inversely associated with participation (Table S1). Intraclass and cross-twin cross-trait correlations were consistently higher for MZ pairs than DZ, implying genetic contributions to all three subscales, and genetic contributions to covariation between SPQ-B subscales (Table S2). Sample characteristics are displayed in Table 1.

\section{INSERT TABLE 1 ABOUT HERE}

\subsection{Association between schizotypy and suicidal ideation at age 22}

At age 22, there was strong evidence that all SPQ-B subscales and total SPQ-B score were associated with suicide ideation when adjusted for age and sex (Table 2). In conditional logistic regression analyses in DZ twin pairs discordant for suicide ideation at age 22, all SPQ-B total and subscale scores were associated with suicidal ideation. In MZ twin pairs, although statistically non-significant, the effect size of the estimate suggested that the negative SPQ-B subscale score was associated with suicidal ideation, and the size of this effect was consistent with the effect in the individual level and within-DZ-pairs analysis. SPQ-B total, positive, and disorganisation scores were associated with suicidal ideation in within-DZ-pair comparisons, but the size of the effect dropped in within-MZ-pair comparisons and there was no longer robust evidence of an association. Results did not differ significantly when analyses were repeated with any reported ideation as an outcome (Table S3), though the relationship between negative schizotypy and suicidal ideation in within-MZ 
pair comparisons became statistically significant and the size of the effect was larger than in the relationship with age 22 suicidal ideation only.

\subsection{Association between suicidal ideation at age 14 and schizotypy at age 22}

In linear fixed effects regression of SPQ-B at age 22 on suicide ideation at age 14, suicide ideation at age 14 was a significant predictor of SPQ-B total, negative, and disorganised scores at the individual level (Table 3). There was marginal evidence that suicidal ideation also predicted positive features. In within-pair comparisons, suicidal ideation did not predict SPQ-B disorganisation in DZ or MZ pairs. The effect size of the associations of suicidal ideation with SPQ-B negative and total scores in within-pair comparisons of $\mathrm{MZ}$ and $\mathrm{DZ}$ pairs were consistent with the individual level associations, and the association of the negative score remained statistically significant within MZ pairs.

INSERT TABLE 2 to 3 ABOUT HERE

\section{Discussion}

We examined the association of schizotypy features and suicidal ideation crosssectionally at age 22 . At the individual level, all schizotypy features were associated with suicidal ideation. As suggested by the effect sizes of the point estimates, negative schizotypy was associated with suicide ideation also within DZ and MZ twin pairs discordant for ideation, suggesting that this relationship is not the result of confounding by shared family environment or genetic factors. In contrast, positive, disorganised, and total schizotypy were associated with suicidal ideation at the individual level, and within DZ pairs, but not within MZ pairs, suggesting shared genetic influences explain the relationship. The temporally reverse association of suicide ideation at age 14 with schizotypy at age 22 was also tested. An association of age 14 suicidal ideation with age 22 negative and total schizotypy was found at 
the individual level, and point estimates were consistent across within-pair analyses of DZ and MZ pairs.

Within-pair analysis of the association of age 22 SPQ-B scores with age 22 suicidal ideation revealed that all SPQ-B subscale scores were significantly associated with suicide ideation at the individual level, and within DZ twin pairs discordant for ideation. This suggests that the schizotypy-suicidal ideation relationship is likely not the result of confounding by shared familial environmental factors, contrary to previous research that has suggested that psychosocial or environmental factors (DeVylder et al., 2015) confound the schizotypy-suicidal ideation relationship.

In MZ pairs discordant for ideation, total, positive, and disorganised schizotypy were not associated with suicide ideation. The relationship of positive (i.e., psychotic experiences) and disorganised aspects of schizotypy with suicidal ideation may therefore result from genetic confounding. Negative schizotypy scores were higher in MZ twins with suicide ideation than in their co-twin without ideation $(O R=1.81,95 \% C I=0.97,3.39)$. The magnitude of this association was consistent with that of the association observed for DZ pairs $(O R=1.46,95 \% C I=1.07,1.98)$ and in the individual level analysis $(O R=1.78,95 \%$ $C I=1.53,2.07)$, supporting an association independent of shared genes. That the finding in MZ pairs did not reach statistical significance, was likely due to reduced statistical power in the smaller sample of MZ pairs discordant for ideation. Importantly, the pattern of associations suggests that negative schizotypy and suicidal ideation are related even when shared environmental and genetic factors are taken into account. The pattern is consistent with there being a causal relationship between schizotypy and suicidal ideation, although confounding by individual-specific environmental factors cannot be ruled out. This result should be treated with caution due to the relatively small sample size of the MZ pair comparison. 
A causal relationship from negative schizotypy to suicidal ideation is consistent with contemporary theoretical models of suicide, such as the interpersonal theory of suicide (IPTS) (Van Orden et al., 2010). In formulating the IPTS, the authors proposed that active suicidal ideation results from a combination of two interpersonal experiences: thwarted belongingness and perceived burdensomeness. It is feasible that negative schizotypy could cause suicidal ideation by increasing the likelihood of thwarted belonging or perceptions of burden, or increasing the degree to which these are experienced. This idea is supported by preliminary evidence that aspects of the schizotypy-suicidal ideation relationship are consistent with some of the assumptions of the IPTS model (Schimanski et al., 2017). That all three components of schizotypy were not uniformly associated with suicidal ideation is consistent with conceptualisations of schizotypy as a heterogeneous construct (Kwapil and Barrantes-Vidal, 2015), and suggests the schizotypy-suicidal ideation could be specific to negative components of schizotypy.

The SPQ-B negative scale has been criticised for largely being composed of social anxiety items (Cohen et al., 2010). If the SPQ-B negative scale is principally a measure of social anxiety, the association of negative schizotypy with suicide ideation may not reflect a causal influence of schizotypy per se. However, cross-twin cross-trait correlations suggested that the SPQ-B negative subscale shares genetic factors with the positive and disorganised subscales (Table S2). This indicates that the negative subscale shares aetiology with the schizotypy construct and is not likely an independent construct.

Tests of reverse association — of the prediction of schizotypy at 22 years from suicidal ideation at 14 years - revealed that suicide ideation was significantly associated with total, negative, and disorganised schizotypy at the individual level, and marginally associated with positive schizotypy. For negative and total scores, point estimates were consistent across individual level, and within-pair analyses for both DZ and MZ pairs, indicating that co-twins 
with suicidal ideation had increased risk for total and negative schizotypy. These findings are consistent with a potential causal relationship from suicidal ideation to negative and total schizotypy. Disorganised schizotypy was not associated with suicidal ideation in within-pair comparisons, suggesting that the ideation-disorganisation relationship is likely due to confounding from shared environmental and genetic factors.

At face value, this pattern suggests reverse causation may affect observed associations between schizotypy and suicidal ideation. However, even with longitudinal designs, it is difficult to distinguish the temporal sequence of schizotypy and suicidal ideation. Schizotypy is regarded as a stable construct (Debbané and Barrantes-Vidal, 2015), not an acquired outcome with a discrete adolescent or early adulthood onset (Meehl, 1990, 1962).

Consequently, schizotypal traits measured at age 22 would be highly correlated with unmeasured schizotypal traits at age 14 and before. Thus, the relationship of age 14 suicidal ideation with age 22 schizotypy in within-pair comparisons of MZ twins, does not necessarily reflect a temporal sequence from which reverse causality can be inferred. Indeed, assuming stability of schizotypy from adolescence through young adulthood, the associations observed in the analyses of reverse causation would also be compatible with a causal relationship from unmeasured schizotypy to suicidal ideation. Despite this limitation, this is the first study to our knowledge to test this reverse association using direct assessment of psychosis features and suicidal ideation, adding to previous research using hospitalisation records (Murphy et al., 2018). That suicidal ideation at age 14 predicts schizotypal indicators eight years later supports the need for further research into this association.

\subsection{Strengths and Limitations}

This study has several strengths. The twin design implicitly controls for genetic and shared familial environment factors without relying on accurate identification and measurement of confounders. Adjustment for confounding factors can generate inaccurate 
estimates of relationships between variables if the factors controlled for are not true confounders (i.e. are a consequence rather than a cause of schizotypy), or there is unmeasured or residual confounding. The twin design avoids these problems through addressing confounding a priori. The protocol included assessment of lifetime suicide ideation, which compared to current ideation is a better predictor of death by suicide (Beck et al., 1999). The predictive value of lifetime ideation may be better as it is an indicator of a latent capacity insofar as the extent to which suicidal ideation has occurred in the past represents the potential for this ideation to become reactivated as part of future processing patterns (Williams et al., 2006). If lifetime suicidal ideation is an accurate predictor of more severe suicidal outcomes this improves the potential for clinical utility of the findings.

Several limitations may affect the interpretation of the study results. First, interpretation of results was limited by the sample size, as there was a relatively small number of MZ twins discordant for ideation $(n=86)$. Second, there are limitations with the use of the SPQ-B due to its psychometric properties (Cohen et al., 2010). There are mixed findings regarding the factor structure of the SPQ-B, with adequate but not unequivocal evidence for a three factor solution (Compton et al., 2009; Fonseca-Pedrero et al., 2017), the forced choice item response has been shown to have lower reliability in SPQ (Wuthrich and Bates, 2005), and the SPQ-B had low to moderate internal consistency in this sample. Further, reliance on self-report could be considered a limitation, though self-report is useful in understanding subjective experiences and observer ratings can introduce additional sources of bias or error (Linscott and van Os, 2013).

Third, suicidal ideation was measured with a single question. It is possible that suicide ideation at age 22 was underreported. In the present sample, $62 \%$ of those who reported suicide ideation at age 14 did not report lifetime suicide ideation at age 22 . Thus, individuals may have been misclassified as having never experienced suicidal ideation at age 22 , when in 
fact they had. However, when analyses were conducted with any reported ideation as an outcome, results did not differ substantially from analyses with age 22 ideation only as an outcome (Table S3). Nevertheless, it is likely there is unmeasured ideation at other timepoints. We also cannot rule out the ideation measure being interpreted differently at different developmental stages, or participants at different ages having different thresholds for responding, introducing measurement error into the results. Lastly, the FinnTwin12 sample is not fully representative of the general population due to oversampling for a parental history of alcohol-related problems. The non-random selection on the basis of familial alcoholism was not associated with any of the suicide or schizotypy variables, except negative schizotypy (Table S1). Although the sample may overrepresent individuals with high negative schizotypy, this would not likely change relationships between variables. Finally, co-twin analyses do not enable control for non-shared, individual-specific environmental risk exposures that may contribute to schizotypy and suicidal ideation. However, as there is no consensus on which factors are true confounders of the schizotypy-suicidal ideation relationship we did not control for any individual-specific factors due to the likelihood they could be mediators, and adjustment would generate inaccurate estimates of relationships.

\subsection{Conclusions}

We found evidence that the relationship between negative schizotypy and suicidal ideation is likely not a result of confounding by shared genetic or common environmental factors. This implies that negative schizotypy and suicide ideation may be causally related, though the possibility of confounding by individual-specific environmental factors cannot be ruled out. Further, the results provide evidence that environmental factors shared between siblings do not explain the relationships of positive or disorganised schizotypy with suicidal ideation, but that their associations may be due to shared genetic factors. Analysis of the reverse association indicated that a reverse causation mechanism, where suicidal ideation 
causes negative schizotypy, could not be ruled out as an explanation. Schizotypy should be considered as a possible causal factor for suicidal ideation, and assessment should consider negative features as well as psychotic experiences. Understanding the pathways linking schizotypy and suicidal ideation is a key focus for future research. 


\section{References}

Allison, P.D., 2009. Fixed Effects Regression Models (Quantitative Applications in the Social Sciences No. 160). Sage Publications Inc., Los Angeles.

Beck, A.T., Brown, G.K., Steer, R.A., Dahlsgaard, K.K., Grisham, J.R., 1999. Suicide ideation at its worst point: a predictor of eventual suicide in psychiatric outpatients. Suicide Life-Threatening Behav. 29, 1-9. https://doi.org/10.1111/j.1943278X.1999.tb00758.x

Brent, D.A., Mann, J.J., 2005. Family Genetic Studies, Suicide, and Suicidal Behavior. Am. J. Med. Genet. Part C Semin. Med. Genet. 24, 13-24. https://doi.org/10.1002/ajmg.c.30042

Bucholz, K.K., Cadoret, R., Cloninger, C.R., Dinwiddie, S.H., Hesselbrock, V.M., Nurnberger, J.I., Reich, T., Schmidt, I., Schuckit, M.A., 1994. A new, semi-structured psychiatric interview for use in genetic linkage studies: a report on the reliability of the SSAGA. J. Stud. Alcohol 55, 149-158. https://doi.org/10.15288/jsa.1994.55.149

Cohen, A.S., Matthews, R.A., Najolia, G.M., Brown, L.A., 2010. Toward a More Psychometrically Sound Brief Measure of Schizotypal Traits: Introducing the SPQ-Brief Revised. J. Pers. Disord. 24, 516-537. https://doi.org/10.1521/pedi.2010.24.4.516

Compton, M.T., Goulding, S.M., Bakeman, R., McClure-Tone, E.B., 2009. An examination of the factorial structure of the Schizotypal Personality Questionnaire-Brief (SPQ-B) among undergraduate students. Schizophr. Res. 115, 286-289. https://doi.org/10.1016/j.schres.2009.04.012

Darvishi, N., Farhadi, M., Haghtalab, T., Poorolajal, J., 2015. Alcohol-related risk of suicidal ideation, suicide attempt, and completed suicide: A meta-analysis. PLoS One 10, 1-14. 
https://doi.org/10.1371/journal.pone.0126870

Debbané, M., Barrantes-Vidal, N., 2015. Schizotypy from a developmental perspective. Schizophr. Bull. 41, S386-S395. https://doi.org/10.1093/schbul/sbu175

DeVylder, J.E., Jahn, D.R., Doherty, T., Wilson, C.S., Wilcox, H.C., Schiffman, J., Hilimire, M.R., 2015. Social and psychological contributions to the co-occurrence of subthreshold psychotic experiences and suicidal behavior. Soc. Psychiatry Psychiatr. Epidemiol. 50, 1819-1830. https://doi.org/10.1007/s00127-015-1139-6

Edenberg, H.J., 2002. The collaborative study on the genetics of alcoholism: an update. Alcohol Res. Health 26, 214-218. https://doi.org/10.1002/ajmg.b.30346.Pooled

Ericson, M., Tuvblad, C., Raine, A., Young-Wolff, K., Baker, L.A., 2011. Heritability and longitudinal stability of schizotypal traits during adolescence. Behav. Genet. 41, 499511. https://doi.org/10.1007/s10519-010-9401-X

Esterberg, M.L., Goulding, S.M., McClure-Tone, E.B., Compton, M.T., 2009. Schizotypy and nicotine, alcohol, and cannabis use in a non-psychiatric sample. Addict. Behav. 34, 374-379. https://doi.org/10.1016/j.addbeh.2008.11.007

Fonseca-Pedrero, E., Ortuño-Sierra, J., Lucas-Molina, B., Debbane', M., Chan, R.C.K., Cicero, D.C., Zhang, L.C., Brenner, C., Barkus, E., Linscott, R.J., Kwapil, T.R., Barrantes-Vidal, N., Cohen, A., Raine, A., Compton, M.T., Tone, E.B., Suhr, J., Bobes, J., Fumero, A., Giakoumaki, S., Tsaousis, I., Preti, A., Chmielewski, M., Laloyaux, J., Mechri, A., Lahmar, M.A., Wuthrich, V., Larøi, F., Badcock, J.C., Jablensky, A., Barron, D., Swami, V., Tran, U.S., Voracek, M., 2017. Brief assessment of schizotypal traits: A multinational study. Schizophr. Res. https://doi.org/10.1016/j.schres.2017.10.043 
Franklin, J.C., Ribeiro, J.D., Fox, K.R., Bentley, K.H., Kleiman, E.M., Huang, X., Musacchio, K.M., Jaroszewski, A.C., Chang, B.P., Nock, M.K., 2017. Risk Factors for Suicidal Thoughts and Behaviors: A Meta-Analysis of 50 Years of Research. Psychol. Bull. 143, 187-232. https://doi.org/10.1037/bul0000084

Glenn, C.R., Cha, C.B., Kleiman, E.M., Nock, M.K., 2017. Understanding Suicide Risk Within the Research Domain Criteria (RDoC) Framework: Insights, Challenges, and Future Research Considerations. Clin. Psychol. Sci. 5, 568-592. https://doi.org/10.1177/2167702616686854

Hawton, K., Saunders, K.E.A., O’Connor, R.C., 2012. Self-harm and suicide in adolescents. Lancet 379, 2373-2382. https://doi.org/10.1016/S0140-6736(12)60322-5

Hielscher, E., DeVylder, J.E., Saha, S., Connell, M., Scott, J.G., 2018. Why are psychotic experiences associated with self-injurious thoughts and behaviours? A systematic review and critical appraisal of potential confounding and mediating factors. Psychol. Med. 48, 1410-1426. https://doi.org/10.1017/S0033291717002677

Honings, S., Drukker, M., Groen, R., Van Os, J., 2016. Psychotic experiences and risk of self-injurious behaviour in the general population: a systematic review and metaanalysis. Psychol. Med. 46, 237-251. https://doi.org/10.1017/S0033291715001841

Jahn, D.R., DeVylder, J.E., Hilimire, M.R., 2016. Explanatory risk factors in the relations between schizotypy and indicators of suicide risk. Psychiatry Res. 238, 68-73. https://doi.org/10.1016/j.psychres.2016.02.021

Jones, H., Heron, J., Hammerton, G., Stochl, J., Jones, P.B., Cannon, M., Davey Smith, G., Holmans, P., Lewis, G., Linden, D.E.J., O’Donovan, M., Owen, M.J., Walters, J.T., 23andMe Research Team, N., Zammit, S., 2018. Investigating the genetic architecture of 
general and specific psychopathology in adolescence. Transl. Psychiatry 8, 145. https://doi.org/10.1038/s41398-018-0204-9

Kaprio, J., 2013. The Finnish Twin Cohort Study: An update. Twin Res. Hum. Genet. 16, 157-162. https://doi.org/10.1017/thg.2012.142

Kaprio, J., 2006. Twin Studies in Finland 2006. Twin Res. Hum. Genet. 9, 772-777. https://doi.org/10.1375/183242706779462778

Kujala, U.M., Kaprio, J., Koskenvuo, M., 2002. Modifiable risk factors as predictors of allcause mortality: The roles of genetics and childhood environment. Am. J. Epidemiol. 156, 985-993. https://doi.org/10.1093/aje/kwf151

Kwapil, T.R., Barrantes-Vidal, N., 2015. Schizotypy: Looking back and moving forward. Schizophr. Bull. 41, S366-S373. https://doi.org/10.1093/schbul/sbu186

Lin, C.C.H., Su, C.H., Kuo, P.H., Hsiao, C.K., Soong, W.T., Chen, W.J., 2007. Genetic and environmental influences on schizotypy among adolescents in Taiwan: A multivariate twin/sibling analysis. Behav. Genet. 37, 334-344. https://doi.org/10.1007/s10519-0069104-5

Linney, Y.M., Murray, R.M., Peters, E.R., MacDonald, A.M., Rijsdijk, F., Sham, P.C., 2003. A quantitative genetic analysis of schizotypal personality traits. Psychol. Med. 33, 803816. https://doi.org/10.1017/S0033291703007906

Linscott, R.J., van Os, J., 2013. An updated and conservative systematic review and metaanalysis of epidemiological evidence on psychotic experiences in children and adults: on the pathway from proneness to persistence to dimensional expression across mental disorders. Psychol. Med. 43, 1133-1149. https://doi.org/10.1017/S0033291712001626 
McGue, M., Osler, M., Christensen, K., 2010. Causal Inference and Observational Research: The Utility of Twins. Perspect. Psychol. Sci. 5, 546-556.

https://doi.org/10.1177/1745691610383511

Meehl, P.E., 1990. Toward an Integrated Theory of Schizotaxia, Schizotypy, and Schizophrenia. J. Pers. Disord. 4, 1-99.

Meehl, P.E., 1971. High school yearbooks: A reply to Schwarz. J. Abnorm. Psychol. 77, 143148. https://doi.org/10.1037/h0030750

Meehl, P.E., 1962. Schizotaxia, schizotypy, schizophrenia. Am. Psychol. 17, 827-838. https://doi.org/10.1037/h0041029

Morton, S.E., O’Hare, K.J.M., Maha, J.L.K., Nicolson, M.P., Machado, L., Topless, R., Merriman, T.R., Linscott, R.J., 2016. Testing the Validity of Taxonic Schizotypy Using Genetic and Environmental Risk Variables. Schizophr. Bull. 43, 633-643. https://doi.org/10.1093/schbul/sbw108

Murphy, J., Shevlin, M., Hyland, P., Christoffersen, M., Elklit, A., Bentall, R., Hyland, P., 2018. Reconsidering the association between psychosis and suicide: a suicidal drive hypothesis. Psychosis 10, 286-297. https://doi.org/10.1080/17522439.2018.1522541

Prinstein, M.J., 2008. Introduction to the special section on suicide and nonsuicidal selfinjury: a review of unique challenges and important directions for self-injury science. $\mathbf{J}$. Consult. Clin. Psychol. 76, 1-8. https://doi.org/10.1037/0022-006X.76.1.1

Raine, A., Benishay, D., 1995. The SPQ-B: A brief screening instrument for schizotypal personality disorder. J. Pers. Disord. 9, 346-355.

Raine, A., Reynolds, C., Lencz, T., Scerbo, A., Triphon, N., Kim, D., 1994. Cognitive- 
perceptual, interpersonal, and disorganized features of schizotypal personality. Schizophr. Bull. 20, 191-200.

Schimanski, I.D., Mouat, K.L., Billinghurst, B.L., Linscott, R.J., 2017. Preliminary evidence that schizophrenia liability at age 15 predicts suicidal ideation two years later. Schizophr. Res. 181, 60-62. https://doi.org/10.1016/j.schres.2016.08.030

Seppä, K., Sillanaukee, P., Koivula, T., 1990. The efficiency of a questionnaire in detecting heavy drinkers. Br. J. Addict. 85, 1639-1645. https://doi.org/10.1111/j.13600443.1990.tb01654.x

Taylor, P.J., Hutton, P., Wood, L., 2015. Are people at risk of psychosis also at risk of suicide and self-harm? A systematic review and meta-analysis. Psychol. Med. 45, 911-926. https://doi.org/10.1017/S0033291714002074

Teraishi, T., Hori, H., Sasayama, D., Matsuo, J., Ogawa, S., Ishida, I., Nagashima, A., Kinoshita, Y., Ota, M., Hattori, K., Kunugi, H., 2014. Relationship between lifetime suicide attempts and schizotypal traits in patients with schizophrenia. PLoS One 9, e107739. https://doi.org/10.1371/journal.pone.0107739

Van Orden, K.A., Witte, T.K., Cukrowicz, K.C., Braithwaite, S.R., Selby, E.A., Joiner, T.E., 2010. The Interpersonal Theory of Suicide. Psychol. Rev. 117, 575-600. https://doi.org/10.1037/a0018697

Williams, J.M.G., Crane, C., Barnhofer, T., Van Der Does, A.J.W., Segal, Z. V., 2006. Recurrence of suicidal ideation across depressive episodes. J. Affect. Disord. 91, 189194. https://doi.org/10.1385/BTER:109:2:189

Williams, R.L., 2000. A note on robust variance estimation for cluster correlated data. Biometrics 56, 645-646. 
World Health Organization, 2014. Preventing suicide. QJM - Mon. J. Assoc. Physicians 97, 677-680. https://doi.org/10.1093/qjmed/hch106

Wuthrich, V., Bates, T.C., 2005. Reliability and validity of two Likert versions of the Schizotypal Personality Questionnaire (SPQ). Pers. Individ. Dif. 38, 1543-1548. https://doi.org/10.1016/j.paid.2004.09.017

Yates, K., Lång, U., Cederlöf, M., Boland, F., Taylor, P., Cannon, M., DeVylder, J.E., Kelleher, I., 2019. Association of psychotic experiences with risk of suicidal ideation, suicide attempts, and suicide deaths: A systematic review and meta-analysis of longitudinal population studies. JAMA psychiatry 76, 180-189. https://doi.org/10.1001/jamapsychiatry.2018.3514 


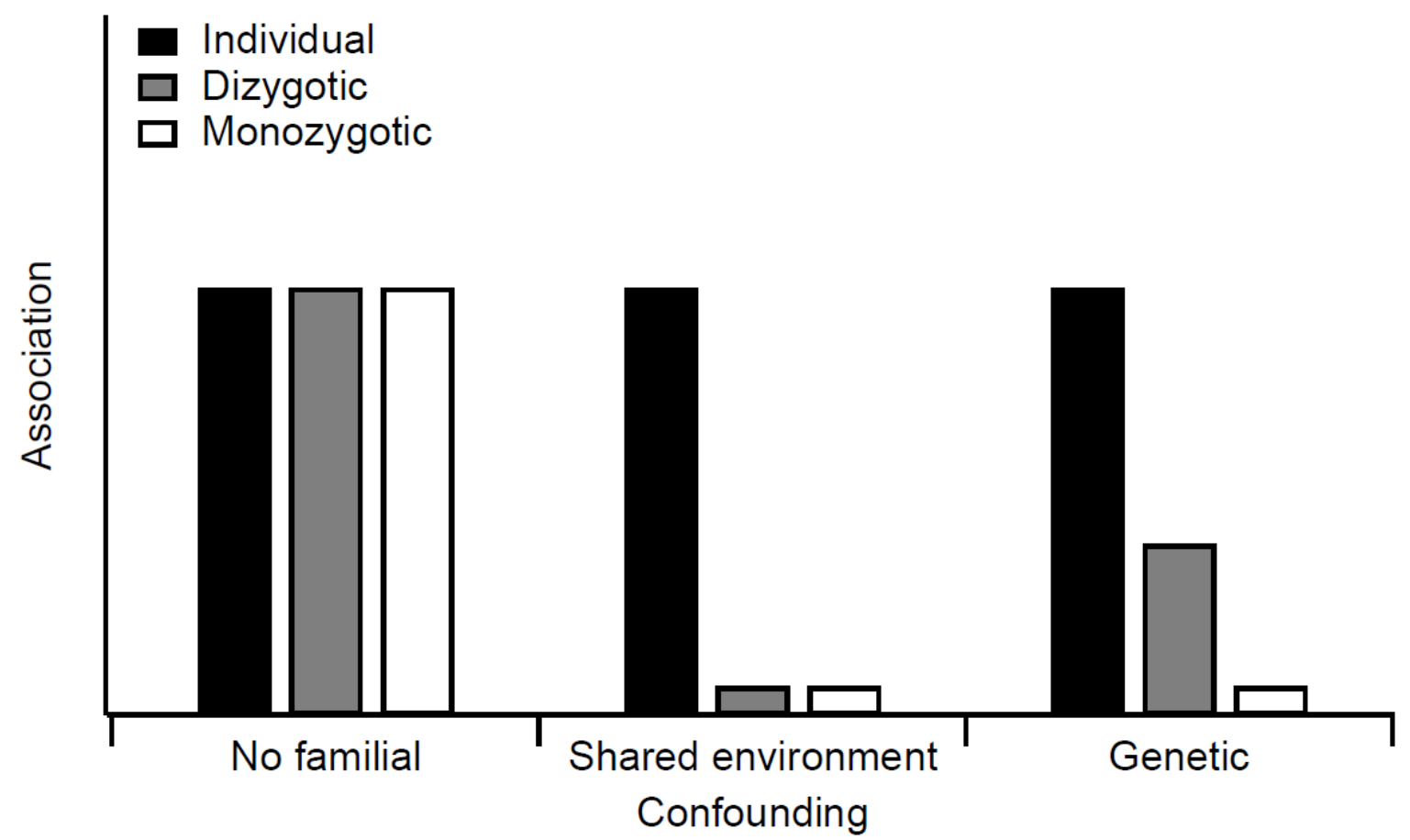

Figure 1. Prototypical patterns of associations in within-twin pair comparisons 
Table 1

Sample Characteristics at Age $14(n=1104)$ and Age $22(n=1213)$ Assessment

\begin{tabular}{|c|c|c|c|c|c|c|c|}
\hline & & $N$ & $\%$ & $M$ & $S D$ & Min & Max \\
\hline Male & & 552 & 45.5 & & & & \\
\hline \multicolumn{8}{|l|}{ Age } \\
\hline & Age 14 interview & & & 14.2 & 0.1 & 14.0 & 14.9 \\
\hline & Age 22 questionnaire & & & 22.4 & 0.7 & 20.5 & 26.5 \\
\hline & Age 22 interview & & & 22.4 & 0.7 & 19.9 & 26.6 \\
\hline
\end{tabular}

Zygosity at age 22

Dizygotic, opposite sex $\quad 338 \quad 27.9$

Dizygotic, same sex $\quad 354 \quad 29.2$

Monozygotic $\quad 520 \quad 42.9$

$\begin{array}{lll}\text { Unknown } & 1 & 0.1\end{array}$

Suicidality outcomes

$\begin{array}{lll}\text { Ideation age 14 } & 81 & 7.7 \\ \text { Ideation age 22 } & 205 & 16.9 \\ \text { Ideation at 14 and 22 } & 31 & 2.9\end{array}$

Schizotypy

SPQ-B Total

$\begin{array}{lllll}5.69 & 4.37 & 0 & 22 & 0.83\end{array}$

SPQ-B Positive

$\begin{array}{lllll}1.75 & 1.71 & 0 & 8 & 0.63\end{array}$

SPQ-B Negative

$\begin{array}{lllll}2.26 & 2.16 & 0 & 8 & 0.78\end{array}$

SPQ-B Disorganised

$\begin{array}{lllll}1.68 & 1.61 & 0 & 6 & 0.68\end{array}$

Note. SPQ-B = Schizotypal Personality Questionnaire-Brief 
Table 2

Prediction of Age 22 Suicidal Ideation from Age 22 SPQ-B Scores in Individual and Within-Pair Analyses

\begin{tabular}{|c|c|c|c|c|c|c|c|c|c|}
\hline & \multicolumn{3}{|c|}{ Individual $^{\mathrm{a}}(n=1213)$} & \multicolumn{3}{|c|}{ Dizygotic twins $^{\mathrm{b}}(n=192)$} & \multicolumn{3}{|c|}{ Monozygotic twins $(n=86)$} \\
\hline & $\mathrm{OR}$ & $95 \% \mathrm{CI}$ & $p$ & $O R$ & $95 \% \mathrm{CI}$ & $p$ & $O R$ & $95 \% \mathrm{CI}$ & $p$ \\
\hline SPQ-B Total & 2.22 & $1.90,2.60$ & $<.001$ & 2.06 & $1.40,3.05$ & $<.001$ & 1.46 & $0.87,2.44$ & .152 \\
\hline SPQ-B Positive & 2.00 & $1.73,2.32$ & $<.001$ & 2.22 & $1.46,3.36$ & $<.001$ & 1.33 & $0.82,2.17$ & .250 \\
\hline SPQ-B Negative & 1.78 & $1.53,2.07$ & $<.001$ & 1.46 & $1.07,1.98$ & .016 & 1.81 & $0.97,3.39$ & .061 \\
\hline SPQ-B Disorganised & 1.87 & $1.61,2.18$ & $<.001$ & 1.77 & $1.23,2.57$ & .002 & 1.06 & $0.66,1.69$ & .824 \\
\hline
\end{tabular}

Note. SPQ-B = Schizotypal Personality Questionnaire-Brief

adjusted for age and sex

badjusted for sex 
Table 3

Prediction of Age 22 SPQ-B Scores from Age 14 Suicide Ideation in Individual and Within-Pair Analyses

\begin{tabular}{|c|c|c|c|c|c|c|c|c|c|}
\hline & \multicolumn{3}{|c|}{ Individual $^{\mathrm{a}}(n=1104)$} & \multicolumn{3}{|c|}{ Dizygotic twins $^{\mathrm{b}}(n=644)$} & \multicolumn{3}{|c|}{ Monozygotic twins $(n=461)$} \\
\hline & $\beta$ & $95 \% \mathrm{CI}$ & $p$ & $\beta$ & $95 \% \mathrm{CI}$ & $p$ & $\beta$ & $95 \% \mathrm{CI}$ & $p$ \\
\hline SPQ-B Total & .36 & $.12, .60$ & .003 & .17 & $-.19, .53$ & .357 & .35 & $-.06, .76$ & .091 \\
\hline SPQ-B Positive & .23 & $-.01, .45$ & .051 & .13 & $-.25, .50$ & .517 & .19 & $-.26, .63$ & .905 \\
\hline SPQ-B Negative & .29 & $.04, .55$ & .025 & .11 & $-.27, .48$ & .577 & .46 & $.03, .89$ & .036 \\
\hline SPQ-B Disorganised & .34 & $.09, .60$ & .008 & .19 & $-.18, .55$ & .322 & .14 & $-.28, .57$ & .511 \\
\hline
\end{tabular}

Note. SPQ-B = Schizotypal Personality Questionnaire-Brief

aadjusted for age and sex

badjusted for sex 